\title{
石墨烯包覆分子篮复合电极材料的制备及其性能研究
}

\author{
赵晓婵 ${ }^{1,2}$, 房艳 ${ }^{1}$, 房春晖 ${ }^{1}$, 周永全 ${ }^{1}$, 戈海文 ${ }^{1}$, 朱发岩 ${ }^{1}$
}

(1. 中国科学院 青海盐湖研究所, 盐湖资源与化学重点实验室, 西宁 810008 ; 2. 中国科学院大学, 北京 100049)

摘 要：以 $4 \mathrm{~A}$ 分子篎(4A)和改进 Hummers 法制备的氧化石墨烯凝胶(GO)为原料，按一定质量比进行混合超声分散， 以混合分散液为前驱体煅烧制备了氧化还原石墨烯(RGO)包覆的三维复合 $4 \mathrm{~A} / \mathrm{RGO}$ 电极材料。采用 $\mathrm{X}$ 射线衍射 $(X R D) 、$ 拉曼光谱(Raman)、孔径分析、扫描电子显微镜(SEM)和电化学测试等方法研究了复合材料的结构、形貌 及超级电容性能。测试结果表明, 4A 均匀地穿插在 RGO 片层中, 阻止了 RGO 片层之间相互堆积, 而 RGO 片层 之间相互链接，形成三维空间导电网络，提高了复合电极材料的导电性。当 GO 与 4A 质量比为 1:6 时，复合材 料在 $4 \mathrm{~A} / \mathrm{g}$ 电流密度下比电容可达 $450 \mathrm{~F} / \mathrm{g}$, 在此电流密度下循环 800 次后, 其比容量保持率为 $85.7 \%$, 表现出良好 的倍率性能和循环稳定性。该 $4 \mathrm{~A} / \mathrm{RGO}$ 复合电极材料超级电容性能优于纯 $4 \mathrm{~A}$ 或 RGO, 可归因于 $4 \mathrm{~A}$ 和 $\mathrm{RGO}$ 之间 的协同效应。

关 键 词: 三维复合电极; 石墨烯; 4A 分子笁; 超级电容器; 协同效应

中图分类号: O646 文献标识码: A

\section{Preparation and Electrochemical Performance of Composite Electrode Material of Molecular Sieve Coated with Graphene}

\author{
ZHAO Xiao-Chan ${ }^{1,2}$, FANG Yan ${ }^{1}$, FANG Chun-Hui ${ }^{1}$, ZHOU Yong-Quan ${ }^{1}$, GE Hai-Wen ${ }^{1}$, ZHU Fa-Yan ${ }^{1}$ \\ (1. Key Laboratory of Salt Lake Resources and Chemistry, Qinghai Institute of Salt Lakes, Chinese Academy of Sciences, \\ Xi ning 810008, China; 2. University of the Chinese Academy of Sciences, Beijing 100049, China)
}

\begin{abstract}
A 3D composite electrode was prepared by ultrasonic dispersion of a suspension mixed proportionally with 4A molecular sieve (4A) and graphene oxide gel (GO). The structure, morphology and electrochemical property of the $4 \mathrm{~A} /$ reduced graphene oxide (RGO) composite were investigated by X-ray diffraction, Raman spectra, pore analysis, scanning electron microscope (SEM) and electrochemical measurements. The result shows that $4 \mathrm{~A}$ is firmly adhered on the surface of RGO sheets, which can effectively avoid the stacking of RGO sheets. The RGO sheets link with each other to form a 3D electric conductive network in which can increase the electrical conductivity of the composite. The specific capacitance of $4 \mathrm{~A} / \mathrm{RGO}$ composite can reach $450 \mathrm{~F} / \mathrm{g}$ at current density of $4 \mathrm{~A} / \mathrm{g}$ when the mass ratio of graphene oxide and $4 \mathrm{~A}$ is $1: 6$. Furthermore, the specific capacitance of $4 \mathrm{~A} / \mathrm{RGO}$ remains at $85.7 \%$ after 800 cycles under the same current density. Therefore these results indicate that this new composite possesses good rate capability and cycle stability and its supercapacitive performance is better than that of pure RGO or $4 \mathrm{~A}$. The excellent performance of the $4 \mathrm{~A} / \mathrm{RGO}$ composite can be attributed to the synergy between RGO and $4 \mathrm{~A}$.
\end{abstract}

Key words: 3D composite; 4A molecular seive; graphene; supercapacitor; synergy 
超级电容器作为一种新型绿色储能器件, 具有 能量密度高、循环寿命长等优点, 在风力发电、存 储备份、军工大功率装备和轨道交通等领域得到广 泛应用 ${ }^{[1-2]}$ 。其中, 电极材料是影响超级电容器电化 学性能的关键因素。目前研究较多的三种超级电容 器电极材料分别为碳材料 ${ }^{[3]}$ 、金属氧化物 ${ }^{[4]}$ 和导电 聚合物 ${ }^{[5]}$ 。研究表明, 将上述不同种类电极材料复合, 产生的协同作用不仅能弥补单一材料不足, 而且能 实现材料性能优势互补。因此, 复合电极材料已成 为超级电容器领域的研究热点。例如, Chan 等 ${ }^{[6]}$ 用电 化学沉积法制备出颗粒状 $\mathrm{MnO}_{2}$ /石墨烯复合物, 并 发现在 $1 \mathrm{mV} / \mathrm{s}$ 扫描速率下其最大比电容为 $378 \mathrm{~F} / \mathrm{g}$ 。 刘建华等 ${ }^{[7]}$ 采用化学接枝法原位合成了石墨烯/聚 吡咯复合物,其比电容可达 $284 \mathrm{~F} / \mathrm{g}$ 。

石墨烯是一种具有蜂窝形状特殊结构的碳材料, 其理论比表面积 $\left(2630 \mathrm{~m}^{2} / \mathrm{g}\right)$ 和电导率 $(7200 \mathrm{~S} / \mathrm{m})$ 远高 于其它碳材料 ${ }^{[8]}$ 。因此, 石墨烯是最具吸引力的电化 学储能材料。但是, 范德华力会使石墨烯发生不可逆 团聚, 从而降低其比表面积和比容量 ${ }^{[9]}$, 单纯石墨烯 电极的超级电容器的实际比容量仅能达到 $200 \mathrm{~F} / \mathrm{g}$ 左 右 ${ }^{[10]}$ 。分子篮虽然具有高的比表面积和均匀的孔隙 率 ${ }^{[11]}$, 但其导电性差, 不能直接用作电极材料。综合 考虑石墨烯和分子笁各自的性能和结构特性, 本工 作拟以分子笁作为填充材料复合到 RGO 层间, 以阻 挡石墨烯片层间的堆叠, 并改善 4A 分子篎的导电性 为基本思路, 制备三维复合 $4 \mathrm{~A} / \mathrm{RGO}$ 电极材料。

\section{1 实验方法}

\section{1 仪器与试剂}

五氧化二磷 $\left(\mathrm{P}_{2} \mathrm{O}_{5}, \mathrm{AR}\right)$ 、过硫酸钾 $\left(\mathrm{K}_{2} \mathrm{~S}_{2} \mathrm{O}_{8}, \mathrm{AR}\right)$ 均为国药试剂, 高锰酸钾 $\left(\mathrm{KMnO}_{4}, \mathrm{AR}\right.$, 天津化学试 剂一厂), 氢氧化钾 $(\mathrm{KOH}, \mathrm{AR}$, 北京化工厂), 无水 乙醇 $\left(\mathrm{C}_{2} \mathrm{H}_{5} \mathrm{OH}, \mathrm{AR}\right.$, 天津大茂化学试剂厂), 过氧化 氢 $\left(\mathrm{H}_{2} \mathrm{O}_{2}, 30 \%\right.$, 天津永大化学试剂厂), 浓硫酸 $\left(\mathrm{H}_{2} \mathrm{SO}_{4}\right.$, 纯度 $>98 \%$, 青海化工科贸试剂厂), 乙炔黑 (纯度 $>98 \%$, Cabot 公司), Nafion 溶液(质量分数 $5 \%$, 上海格式新能源技术有限公司), 鳞片石墨(粒度 $180 \mu \mathrm{m}$, 含碳 $99 \%$, 青岛申墅石墨制品厂), 4A 分子 篮(粒径 180 250 $\mathrm{m} \mathrm{m}$, 孔径 $0.4 \mathrm{~nm}$, 山东罗孚高新 材料有限公司), 泡沫镍(规格: $2 \mathrm{~mm} \times 200 \mathrm{~mm} \times$ $300 \mathrm{~mm}$, 开孔率: 99\%, 青岛天盛达石墨有限公司)。

精科申光 WG-DCZ 型低温恒温槽, PGSTAT128N 型德国万通电化学工作站, 杭州成功 FS-300N 型超声波处理器, 合肥科晶 GSL-1200X 管
式炉和 $24 \mathrm{~T}$ 手动压片机。

\section{2 样品的制备}

采用改进 Hummers 法 ${ }^{[12]}$ 制备 GO, 具体过程如 下: 移取 $40 \mathrm{~mL} 、 98 \mathrm{wt} \%$ 的浓 $\mathrm{H}_{2} \mathrm{SO}_{4}$, 置于 $250 \mathrm{~mL}$ 烧杯中, 加入 $6 \mathrm{~g} \mathrm{~K}_{2} \mathrm{~S}_{2} \mathrm{O}_{8}$ 和 $6 \mathrm{~g} \mathrm{P}_{2} \mathrm{O}_{5}$, 磁力搅拌均匀, 往混合液中加鳞片石墨 $10 \mathrm{~g}$, 冰水浴 $0^{\circ} \mathrm{C}$ 搅拌, $75^{\circ} \mathrm{C}$ 反应 $5 \mathrm{~h}$, 冷却至室温, 静置过夜, 过滤上清液, 然 后 $45^{\circ} \mathrm{C}$ 烘干。取 $2 \mathrm{~g}$ 上述预氧化石墨粉加入 $100 \mathrm{~mL}$ 浓 $\mathrm{H}_{2} \mathrm{SO}_{4}$, 冰水浴 $0^{\circ} \mathrm{C}$ 搅拌, 加入 $20 \mathrm{~g} \mathrm{KMnO}_{4}$, 继续 搅拌 $20 \mathrm{~min}$, 然后 $40^{\circ} \mathrm{C}$ 反应 $4 \mathrm{~h}$, 最后倒入 $1000 \mathrm{~mL}$ 蒸馏水, 再逐滴滴加 $30 \mathrm{~mL} \mathrm{H}_{2} \mathrm{O}_{2}$, 溶液反应至呈现 金黄色即可。最后, 将溶液离心, 透析洗涤沉淀至中 性, 产物为 $\mathrm{GO}$ 。

称 $4 \mathrm{~A}(1.5 \mathrm{~g})$ 置于 $250 \mathrm{~mL}$ 烧杯中, 加入 $50 \mathrm{~mL}$ 分散好的 $\mathrm{GO}(5 \mathrm{mg} / \mathrm{mL})$ 凝胶, 超声溶解 10 15 min 后, 强力搅拌 $30 \mathrm{~min}$, 得到均匀悬浮液。悬浮液 $80^{\circ} \mathrm{C}$ 不 断搅拌蒸发水分, 将所得糊状物置于烘箱中 $45^{\circ} \mathrm{C}$ 烘 干, 在氮气保护条件下 $320^{\circ} \mathrm{C}$ 灼烧 $2 \mathrm{~h}$, 得到的产物 自然冷却至室温, 即得 $4 \mathrm{~A} / \mathrm{RGO}$ 复合电极材料。

\section{3 制备电极}

泡沫镍为集流体, 用丙酮、乙醇及蒸馏水依次 超声清洗泡沫镍, 真空烘干待用。将 $4 \mathrm{~A} / \mathrm{RGO}$ 、乙 炔黑和 Nafion 溶液按 $8: 1: 1$ 质量比, 加入适量乙 醇超声分散, $60^{\circ} \mathrm{C}$ 磁力摚拌成塑性浆料, 涂在清洗 好的泡沫镍上, 压片机以 $10 \mathrm{MPa}$ 压力保持 $2 \mathrm{~min}$ 压 成薄片电极, $45^{\circ} \mathrm{C}$ 真空干燥 $12 \mathrm{~h}$, 得 $4 \mathrm{~A} / \mathrm{RGO}$ 电极。

为了比较纯 $4 \mathrm{~A}$ 或 $\mathrm{RGO}$ 电极与 $4 \mathrm{~A} / \mathrm{RGO}$ 电极差 异, 在相同条件下, 制备纯 $4 \mathrm{~A}$ 和 RGO 电极。

\section{4 样品表征}

\subsection{1 物理性能测试}

采用荷兰 PANalytical 公司 $\chi$ 'Pert Pro 型 X 射线 衍射仪, 选择 $\mathrm{Cu}$ 靶, $\mathrm{K} \alpha$ 辐射 $(\lambda=0.154060 \mathrm{~nm})$, 石墨 单色器滤波, 管压为 $40 \mathrm{kV}$, 管电流为 $40 \mathrm{~mA}, 2 \theta$ 角 范围为 $0^{\circ} \sim 80^{\circ}$, 粉末 $\mathrm{X}$ 射线衍射, 将样品研磨后进 行 XRD 测试。Raman 测试采用美国 Thermo Scientific 公司 DXR 型拉曼光谱仪(激发波长 $455 \mathrm{~nm}$ 、功 率 $6 \mathrm{~mW}$ 、曝光时间 $20 \mathrm{~s}$ 、波数范围 100 4000 $\mathrm{cm}^{-1}$ )。 采用美国康塔公司 Autosorb $\mathrm{iQ}_{2}$ 型孔径分析仪, 测 试温度为 $77.3 \mathrm{~K}$, 相对压力范围为 $0 \sim 1$, BJH 方法计 算孔径分布。采用日本电子株式会社英国牛津仪器 公司低真空扫描电子显微镜 JSM-5610LV/INCA 观 察样品形貌。

\subsection{2 电化学性能测试}

采用标准三电极体系进行电化学性能测试, 石 
墨棒 $(\phi 6 \mathrm{~mm})$ 和录-氧化永电极分别为对电极和参比 电极。所有电化学性能测试在 AUTOLAB PGSTAT $128 \mathrm{~N}$ 型电化学工作站上完成: 在-1.2 0.2 V 电位区 间进行循环伏安法测试; 恒电流充放电测试电位范 围为-1.2 0.2 $\mathrm{V}$ (纯 $4 \mathrm{~A}$ 恒电流充放电测试电压区间 为 $-0.3 \sim 0.6 \mathrm{~V}$ ); 电化学交流阻抗 (EIS) 测试频率 $0.01 \sim 100 \mathrm{kHz}$, 振幅 $0.01 \mathrm{~V}$ 。测试过程用低温恒温槽 控制测试温度在 $(25.0 \pm 0.1)^{\circ} \mathrm{C}$ 。

\section{2 结果与讨论}

\section{1 结构分析}

图 1(a)为 $\mathrm{GO} 、 \mathrm{RGO} 、 4 \mathrm{~A}$ 和 $4 \mathrm{~A} / \mathrm{RGO}$ 的 XRD 图谱。GO在 $2 \theta=10.9^{\circ}$ 处有一个尖锐的强衍射峰, 归 属于 $\mathrm{GO}(100)$ 晶面衍射, 其峰型规整, 峰宽较窄, 表 明其具有良好 $\mathrm{GO}$ 结构 ${ }^{[13]}$ 。当 $\mathrm{GO}$ 被还原为 $\mathrm{RGO}$ 时, 在 $2 \theta=23.5^{\circ}$ 附近存在宽化弱衍射峰, 因 RGO 粒 径过小, 表面上残存的羟基易与边缘含氧基团结合 形成絮凝体, 从而形成无序堆积 $\mathrm{RGO}$ 薄膜, 在 XRD 测试中无明显衍射峰。 $4 \mathrm{~A} / \mathrm{RGO}$ 衍射峰同 $4 \mathrm{~A}$ 衍射峰位置基本一致, 说明加入 $\mathrm{RGO}$ 并没有改变 $4 \mathrm{~A}$ 自身结构。由于 $\mathrm{RGO}$ 添加量较小, 且碳衍射能 力较弱, 所以 $\mathrm{RGO}$ 衍射峰被 $4 \mathrm{~A}$ 衍射峰覆盖, 复合 材料图谱中并未出现 $\mathrm{RGO}$ 特征衍射峰。

图 1(b)为 RGO、4A 和 4A/RGO 的 Raman 谱图, 可以看出 $\mathrm{RGO}$ 在 $1369 、 1599 \mathrm{~cm}^{-1}$ 处各有一个宽峰, 对应 RGO 的 D 和 G 峰, D 峰由 RGO 表面缺陷和其 原子无序度产生, $\mathrm{G}$ 峰由 $\mathrm{RGO}$ 片层碳环中 $\mathrm{sp}^{2}$ 原子 对拉伸运动产生。一般 $\mathrm{D}$ 和 $\mathrm{G}$ 峰强度比 $\left(I_{\mathrm{D}} / I_{\mathrm{G}}\right)$ 与石 墨微晶尺寸大小相关, 可衡量碳材料无序度或缺陷 密度 ${ }^{[14]}$, 图 1(b)中 $\mathrm{RGO}$ 的 $I_{\mathrm{D}} / I_{\mathrm{G}}$ 值为 0.74 , 说明 $\mathrm{RGO}$ 边缘缺陷、空穴等较低, 是一种质量较好的碳材料。 $4 \mathrm{~A} / \mathrm{RGO}$ 复合电极材料中也存在 $\mathrm{RGO}$ 的 $\mathrm{D}$ 和 $\mathrm{G}$ 峰, 它的 $I_{\mathrm{D}} / I_{\mathrm{G}}$ 值为 1.1 , 说明 $\mathrm{RGO}$ 包覆 $4 \mathrm{~A}$ 之后, $\mathrm{RGO}$
片层碳环中 $\mathrm{sp}^{2}$ 原子对相对位置发生变化, 即 RGO 孔径发生了变化。同时, $488 \mathrm{~cm}^{-1}$ 处 $4 \mathrm{~A}$ 分子篎的特 征峰很弱, 说明是 RGO 包覆了 $4 \mathrm{~A}$ 。

由图 1(a)和(b)结果可以得出, 复合材料中 $R G O$ 包覆了 $4 \mathrm{~A}$, 包覆之后 $\mathrm{RGO}$ 的结构发生了改变, 而 $4 \mathrm{~A}(0.4 \mathrm{~nm})$ 仍保持其原有结构, $4 \mathrm{~A}$ 的特殊孔径可使 电解液中阴阳离子自由穿越表层的 RGO 层, 形成 三维导电网络结构, 从而有利于电荷在电极表面的 运输与存储。

\section{2 孔径分析}

图 2 是 $\mathrm{RGO}$ 与 $4 \mathrm{~A} / \mathrm{RGO}$ 孔径分布图，由图可以 看出, 与纯 $\mathrm{RGO}$ 相比, $4 \mathrm{~A} / \mathrm{RGO}$ 孔径在 $0.16 \sim 0.2 \mathrm{~nm}$ 之间的比例明显增加, 此结果验证了 Raman 的结论, 即 RGO 包覆 4A 之后其结构发生了变化。研究发现, 电解液中阴阳离子直径与电极材料孔径越接近, 电 极表面单位面积电荷存储量越多, 超级电容器比电 容也就越大 ${ }^{[15]}$ 。例如, Chmiola 等 ${ }^{[16]}$ 通过缩小活性材 料孔径 $(<2 \mathrm{~nm})$, 使电极材料的比电容在此孔径范围 内明显提高, 这一结论已经得到理论分析证实 ${ }^{[17]}$ 。

图 2 给出电解液中阴阳离子 $\left(\mathrm{OH}^{-} 、 \mathrm{~K}^{+}\right)$的直径均小于 $0.3 \mathrm{~nm}$,包覆 $4 \mathrm{~A}$ 之后的 $\mathrm{RGO}$ 孔径与电解液中阴阳离 子直径大小更接近, 结果说明包覆了 $4 \mathrm{~A}$ 后的 $\mathrm{RGO}$ 层, 更有利于电极表面正负电荷的运输与存储。

\section{3 形貌分析}

图 3 为电极材料的 SEM 照片, 图 3(a)、(b)中 $\mathrm{RGO}$ 片之间交织成多孔网状结构, RGO 中含氧官能 团减少，导致 RGO 之间由于范德华力作用易团聚， 片层间堆叠很明显。图 3(c)、(d)中 $4 \mathrm{~A}$ 晶体结构呈 现明显立方晶系结晶。图 3(e)、(f)中 RGO 包覆在 $4 \mathrm{~A}$ 表面, $4 \mathrm{~A}$ 均匀地穿插在 $\mathrm{RGO}$ 片层中, 阻止了 $\mathrm{RGO}$ 片层之间相互堆叠, 电解液中的阴阳离子可以 自由地穿梭在 $4 \mathrm{~A}$ 内部与表层的 RGO, 形成三维空 间导电网络, 提高复合电极的电子导电性。由于 RGO 片大小不均匀, 较小的片层紧贴在微球表面,

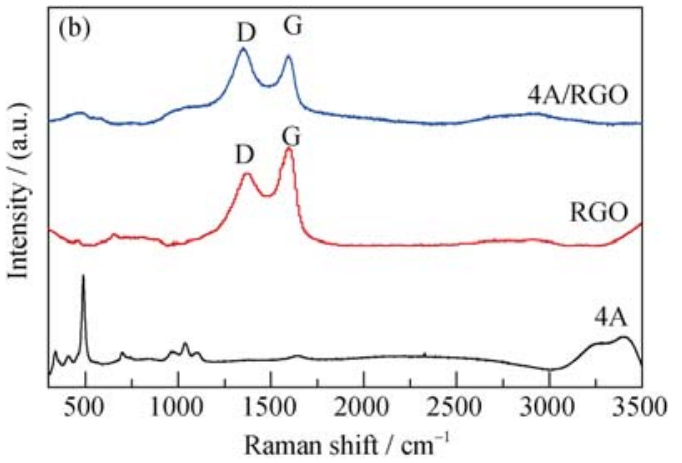

图 1 样品的 XRD 图谱 (a)和 Raman 谱图(b)

Fig. 1 XRD patterns (a) and Raman spectra (b) of samples 


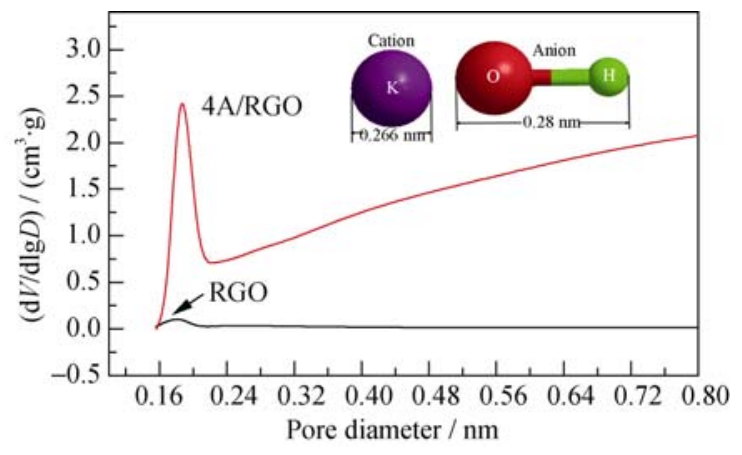

图 $2 \mathrm{RGO}$ 和 $4 \mathrm{~A} / \mathrm{RGO}$ 的孔径分布图

Fig. 2 Pore size distribution of RGO and 4A/RGO

而较大的 RGO 片可将微球完全包覆, 该复合电极 材料结构属于封装型 ${ }^{[18-19]}$ 。

\section{4 电化学性能分析}

\subsection{1 循环伏安测试}

图 4(a)为电极材料在 $10 \mathrm{mV} / \mathrm{s}$ 扫描速率下的循
环伏安曲线 ( $\mathrm{CV}$ 曲线)。 $\mathrm{RGO}$ 与 $4 \mathrm{~A} / \mathrm{RGO}$ 的 $\mathrm{CV}$ 曲 线形状相似, 都有一对较弱的氧化还原峰, 是由 $\mathrm{RGO}$ 上含氧官能团氧化还原反应引起的。在相同电 位窗口下, $4 \mathrm{~A} / \mathrm{RGO}$ 比 RGO 的电流明显增大, $\mathrm{CV}$ 曲 线面积也随之增大，表明 $4 \mathrm{~A} / \mathrm{RGO}$ 复合电极材料的 比电容高于 $\mathrm{RGO}$ 。图 4(b) 是 $4 \mathrm{~A} / \mathrm{RGO}$ 复合电极材料 在 $6 \mathrm{~mol} / \mathrm{L} \mathrm{KOH}$ 电解液中不同扫速下的 $\mathrm{CV}$ 曲线, 曲线对称性良好，没有明显氧化还原峰，说明材料 结构稳定 ${ }^{[19]}$ 。

\subsection{2 充放电测试}

图 5 为电极材料在 $6 \mathrm{~mol} / \mathrm{L} \mathrm{KOH}$ 电解液中测得 的恒电流充放电曲线(Galvanostatic charge-discharge, $\mathrm{GCD})$ 。比电容可通过公式 $(C=I \Delta t / m \Delta V$ 计算得到 (其中 $C$ 为比电容 $(\mathrm{F} / \mathrm{g}) ; I$ 为充放电电流 $(\mathrm{A}) ; \Delta t$ 为放 电时间 (s); $m$ 为活性物质质量 $(\mathrm{g}) ; \Delta V$ 为循环伏安电 势窗口 $(\mathrm{V}))$ 。图 5(a)为不同电流密度下 $4 \mathrm{~A} / \mathrm{RGO}$ 复 合电极材料 $\mathrm{GCD}$ 曲线, 由图可见 $4 \mathrm{~A} / \mathrm{RGO}$ 复合电
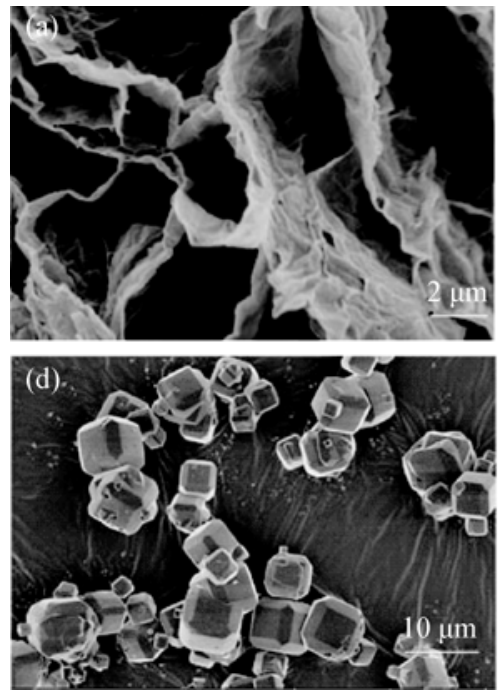
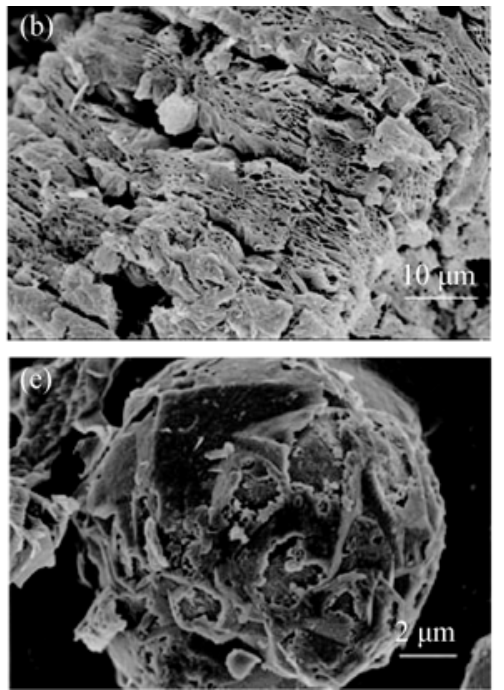
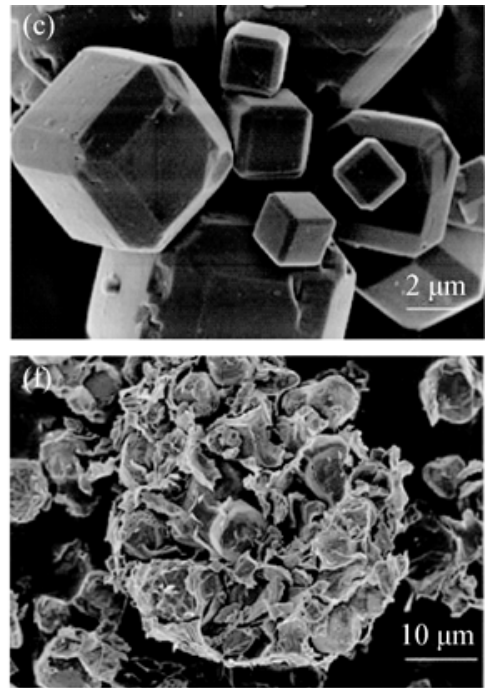

图 $3 \mathrm{RGO}(\mathrm{a} 、 \mathrm{~b}) 、 4 \mathrm{~A}(\mathrm{c} 、 \mathrm{~d})$ 及 $4 \mathrm{~A} / \mathrm{RGO}(\mathrm{e} 、 \mathrm{f})$ 的 SEM 照片

Fig. 3 SEM images of RGO (a, b),4A (c, d) and 4A/RGO (e, f)
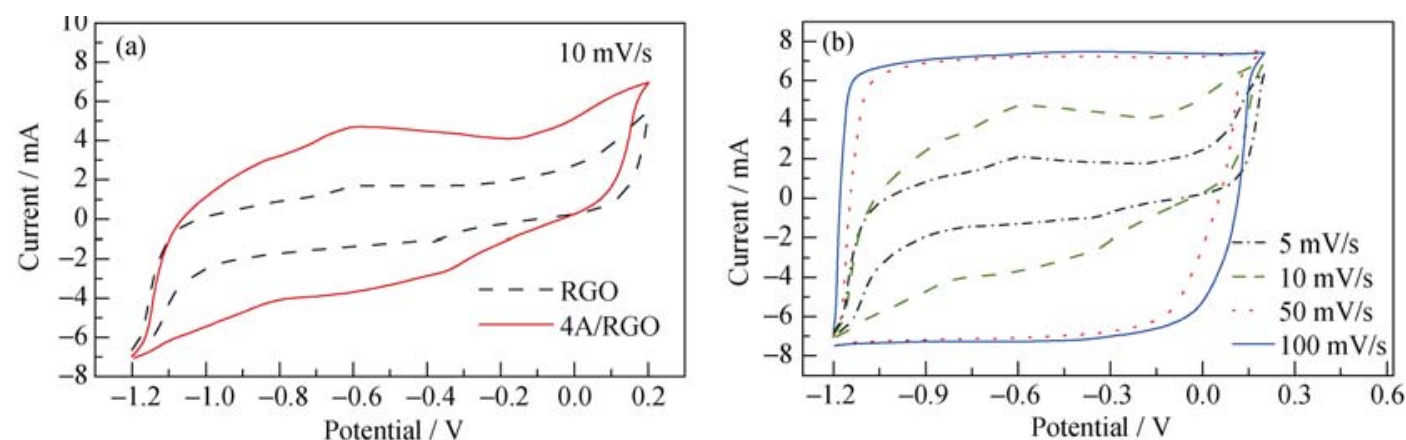

图 4 (a)RGO 和 $4 \mathrm{~A} / \mathrm{RGO}$ 复合电极材料在 $10 \mathrm{mV} / \mathrm{s}$ 扫描速率时的 $\mathrm{CV}$ 曲线和(b) $4 \mathrm{~A} / \mathrm{RGO}$ 复合电极材料在 $6 \mathrm{~mol} / \mathrm{L} \mathrm{KOH}$ 中不同扫 速时的 CV 曲线

Fig. 4 (a) CV curves of RGO, and 4A/RGO composite at scanning rate of $10 \mathrm{mV} / \mathrm{s}$ and (b) $\mathrm{CV}$ curves of $4 \mathrm{~A} / \mathrm{RGO}$ composite at various scan rates in $6 \mathrm{~mol} / \mathrm{L} \mathrm{KOH}$ solution 
极材料在不同电流密度下都表现出良好的充放电特 性。随着电流密度的增加, 复合电极的比电容值降 低, 但电流密度为 $12 \mathrm{~A} / \mathrm{g}$ 时, $4 \mathrm{~A} / \mathrm{RGO}$ 复合电极材料 的比电容值仍保持在 $421 \mathrm{~F} / \mathrm{g}$, 说明 $4 \mathrm{~A} / \mathrm{RGO}$ 具有良 好的倍率特性。

图 5(b)为 $4 \mathrm{~A} / \mathrm{RGO}$ 复合电极材料在电流密度为 $4 \mathrm{~A} / \mathrm{g}$ 下前 10 次 $\mathrm{GCD}$ 曲线。 $4 \mathrm{~A} / \mathrm{RGO}$ 的 GCD 曲线 呈三角形对称分布，电压与时间基本呈线性关系， $\mathrm{GCD}$ 曲线的斜率 $(\mathrm{d} V / \mathrm{d} t)$ 基本保持恒定, 循环性能很 好, 说明 $4 \mathrm{~A} / \mathrm{RGO}$ 具有良好的电化学可逆性和充放 电循环性能, 展示其良好电容性能。纯 RGO 的 GCD 曲线存在着较明显的电压降, 而 $4 \mathrm{~A} / \mathrm{RGO}$ 电极材料 具有三角对称性, 且无明显电压降存在, 表现出良 好的电化学可逆性 ${ }^{[20]}$ 。由公式可计算材料比容量 ${ }^{[21]}$, 纯 $4 \mathrm{~A} 、 \mathrm{RGO}$ 和 $4 \mathrm{~A} / \mathrm{RGO}$ 电极材料在 $4 \mathrm{~A} / \mathrm{g}$ 电流密 度下比电容分别为 $18 、 101$ 和 $450 \mathrm{~F} / \mathrm{g}, 4 \mathrm{~A} / \mathrm{RGO}$ 的 比容量远大于纯 RGO 和 $4 \mathrm{~A}$ 。

图 5(d)为电极材料在 4 16 A/g 电流密度下根据 公式计算的比电容曲线。所有电极材料比电容均随 电流密度升高而降低, 其中, $4 \mathrm{~A}$ 电极的比电容降低 较快, 且电流密度在 $10 \mathrm{~A} / \mathrm{g}$ 以上时无比电容; 而 $\mathrm{RGO}$ 和 $4 \mathrm{~A} / \mathrm{RGO}$ 即使在大电流密度 $(12 \mathrm{~A} / \mathrm{g})$ 下比电 容仍可达 79 和 $421 \mathrm{~F} / \mathrm{g}$, 表现出了良好倍率特性。 这是由于纯 $4 \mathrm{~A}$ 电极材料导电性能差, 所以其电化

学性能不及 $4 \mathrm{~A} / \mathrm{RGO}$ 。而 $4 \mathrm{~A} / \mathrm{RGO}$ 的电化学性能强 于纯 $R G O$ 是由于包覆了 $4 \mathrm{~A}$ 的 $\mathrm{RGO}$ 形成三维网络 导电结构, 阻止了 RGO 的团聚, 这种结构更有利于 正负电荷在电极材料表面传输与积累。所以, $4 \mathrm{~A} / \mathrm{RGO}$ 复合电极材料超级电容性能优于纯 $4 \mathrm{~A}$ 或 $\mathrm{RGO}$, 可归于 $4 \mathrm{~A}$ 和 $\mathrm{RGO}$ 之间的协同效应。

\subsection{3 交流阻抗测试}

图 6 为电极材料在 $6 \mathrm{~mol} / \mathrm{L} \mathrm{KOH}$ 电解液中, 三电 极体系下测得的电化学交流阻抗复数平面(Nyquist) 图, 高频 $10^{5} \mathrm{~Hz}$, 低频 $0.01 \mathrm{~Hz}$, 振幅 $0.01 \mathrm{~V}$ 。所有电 极材料曲线均由高频区半圆弧和低频区直线组成。 高频区半圆弧的出现是由于电解质溶液中离子在电 极材料上的电荷运输存储, 产生的电荷转移电阻, 即高频区半圆弧大小决定电解液/电极材料电极界 面的电荷传输反应引起阻抗 $\left(R_{\mathrm{ct}}\right)$ 的大小。由图 6 高 频区放大图可以看出, $4 \mathrm{~A} / \mathrm{RGO}$ 半圆直径小于纯 $4 \mathrm{~A}$ 和 $\mathrm{RGO}$ 半圆直径, 半圆直径越小, 电极电荷转移电 阻越小, 即 $4 \mathrm{~A} / \mathrm{RGO}$ 导电性能越好 ${ }^{[22]}$ 。低频区直线 代表 Warburg 阻抗 $Z_{\mathrm{w}}$ ，一般而言，在交流阻抗图中， 低频区直线倾角越接近于 $90^{\circ}$, 说明材料电容特性 越好。4A/RGO 阻抗曲线在低频区几乎垂直于横轴, 说明电解液中阴阳离子传输过程中, 正负电荷在 $4 \mathrm{~A} / \mathrm{RGO}$ 电极表面受到阻力最小, 即三种材料中 $4 \mathrm{~A} / \mathrm{RGO}$ 导电性能最佳。
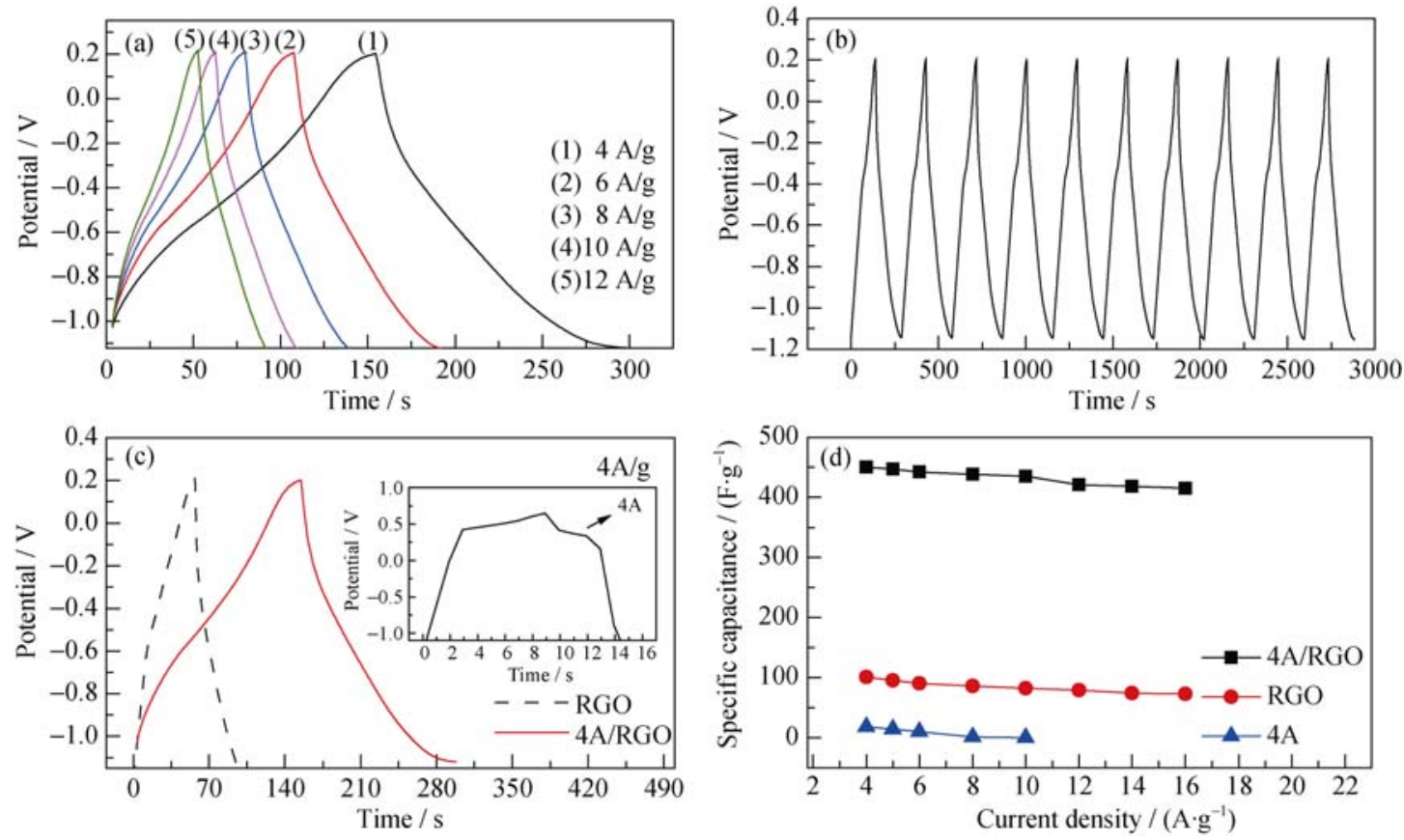

图 $5 \mathrm{RGO} 、 4 \mathrm{~A}$ 和 $4 \mathrm{~A} / \mathrm{RGO}$ 的恒电流充放电测试结果

Fig. 5 Constant current charge and discharge curves of $4 \mathrm{~A}, \mathrm{RGO}$ and $4 \mathrm{~A} / \mathrm{RGO}$

GCD curves of the materials tested in the $6 \mathrm{~mol} / \mathrm{L} \mathrm{KOH}$; (b) GCD curves (1-10th) of 4A/RGO composites at current density of $4 \mathrm{~A} / \mathrm{g}$; (c) Comparison of GCD curves with RGO and $4 \mathrm{~A} / \mathrm{RGO}$ at current density of $4 \mathrm{~A} / \mathrm{g}$; and (d) Specific capacitance of different materials at current density from $4 \mathrm{~A} / \mathrm{g}$ to $16 \mathrm{~A} / \mathrm{g}$ 


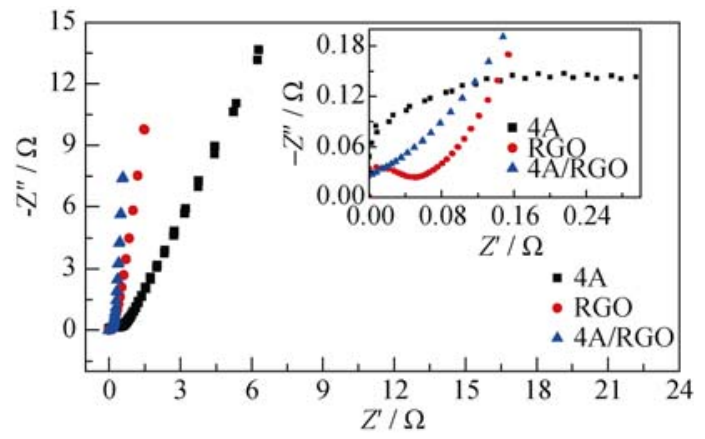

图 $6 \mathrm{RGO} 、 4 \mathrm{~A}$ 和 $4 \mathrm{~A} / \mathrm{RGO}$ 在 $6 \mathrm{~mol} / \mathrm{L} \mathrm{KOH}$ 溶液中的 $\mathrm{EIS}$ 曲线 Fig. 6 EIS curves of RGO, 4A and 4A/RGO composite in $6 \mathrm{~mol} / \mathrm{L} \mathrm{KOH}$ solution

\subsection{4 循环性能测试}

图 7 是电极材料在 $4 \mathrm{~A} / \mathrm{g}$ 电流密度下的充放电 循环寿命图, 可看到 800 次充放电后 $4 \mathrm{~A} / \mathrm{RGO} 、 \mathrm{RGO}$ 和纯 $4 \mathrm{~A}$ 的电容保持率分别为 $85.7 \%$ 、 $75.3 \%$ 和 $63.3 \%$ 。 RGO 作为电极材料其电容稳定性较好, 而 4A 电容稳定性远低于碳材料稳定性。当两者结合成 复合电极材料后, 其电容稳定性得到显著提高。分 子篮的加入能够提高电极材料稳定性, 这是由于复 合电极材料中 $4 \mathrm{~A}$ 和 RGO 紧密结合以及多微孔结构 提供了电解液离子在充放电时的传输通道, 两者之 间产生协同作用从而保持较高稳定性。

\section{3 结论}

以 $4 \mathrm{~A}$ 分子篮和 $\mathrm{GO}$ 为原料, 制备出新型 $\mathrm{RGO}$ 包覆的三维复合 $4 \mathrm{~A} / \mathrm{RGO}$ 电极材料, RGO 可将 $4 \mathrm{~A}$ 完全包覆, 且 $4 \mathrm{~A}$ 均匀地穿插在 RGO 片层中, 阻止 RGO 片层之间相互堆积, 电解液中的阴阳离子可以 自由穿梭在 $4 \mathrm{~A}$ 内部与 $\mathrm{RGO}$ 层, 形成三维空间导电 网络。电化学性能测试表明, 在电流密度为 $4 \mathrm{~A} / \mathrm{g}$, $\mathrm{GO}$ 与 $4 \mathrm{~A}$ 质量比 1:6 条件下, $4 \mathrm{~A} / \mathrm{RGO}$ 电极材料的 比电容为 $450 \mathrm{~F} / \mathrm{g}$, 远高于纯 $4 \mathrm{~A}(18 \mathrm{~F} / \mathrm{g})$ 和 $\mathrm{RGO}(101 \mathrm{~F} / \mathrm{g})$ 。在此条件下经过 800 次循环, $4 \mathrm{~A} / \mathrm{RGO}$ 电极材料的比电容仍保持最高比电容的 $85.7 \%$ 。本文报道的三维复合 $4 \mathrm{~A} / \mathrm{RGO}$ 电极材料制 备方法简便, 该材料有望应用于大规模制备, 是一 种有潜在应用价值的超级电容器电极材料。

\section{参考文献:}

[1] LIU J, WICKRAMARATENE N P, QIAO S Z, et al. Molecularbased design and emerging applications of nanoporous carbon spheres. Nature Materials, 2015, 14(8): 763-774.

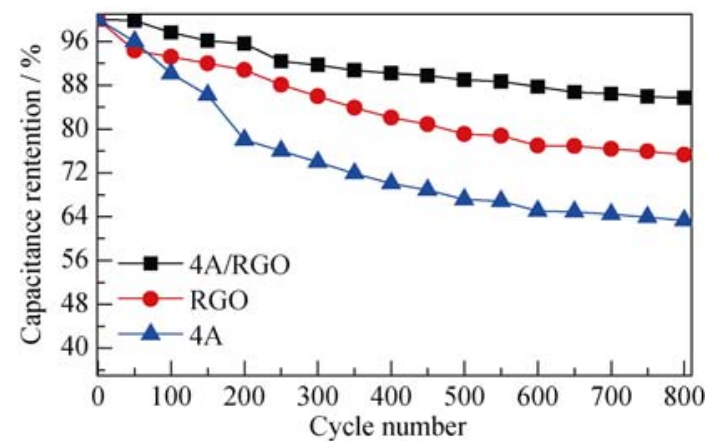

图 $74 \mathrm{~A} / \mathrm{g}$ 电流密度下 RGO、 $4 \mathrm{~A}$ 和 $4 \mathrm{~A} / \mathrm{RGO}$ 的循环稳定性 Fig. 7 Cycle stability of RGO, 4A and $4 \mathrm{~A} / \mathrm{RGO}$ at current density of $4 \mathrm{~A} / \mathrm{g}$

\section{参考文献:}

[2] SHAO Y, EI-KADY M F, WANG L J, et al. Graphene-based materials for flexible supercapacitors. Chemical Society Reviews, 2015, 44(11): 3639-3665.

[3] WANG L, TIAN L H, YU G D, et al. Epitaxial growth of graphene and their applications in devices. Journal of Inorganic Materials, 2011, 10: 1009-1019.

[4] CHEN S, ZHU J, WU X, et al. Graphene oxide- $\mathrm{MnO}_{2}$ nanocomposites for supercapacitors. ACS Nano, 2010, 4(5): 2822-2830.

[5] LIU D Y, REYNOLDS J R. Dioxythiophene-based polymer electrodes for supercapacitor modules. ACS Applied Materials \& Interfaces, 2010, 2(12): 3586-3593.

[6] CHAN P Y, MAJID S R. RGO-wrapped $\mathrm{MnO}_{2}$ composite electrode for supercapacitor application. Solid State Ionics, 2014, 262: 226-229.

[7] LIU J H, ZHANG S L, YU M, et al. Synthesis and capacitance characteristics of the graphene grafted polypyrrole composites. Journal of Inorganic Materials, 2013, 04: 403-408.

[8] SINGH V, JOUNG D, ZHAI L, et al. Graphene based materials: past, present and future. Progress in Materials Science, 2011, 56(8): $1178-1271$.

[9] GEORGAKILAS V, OTYEPKA M, BOURLIONS A B, et al. Functionalization of graphene: covalent and non-covalent approaches, derivatives and applications. Chemical Reviews, 2012, 112(11): 6156-6214.

[10] STOLLER M D, PARK S, ZHU Y W, et al. Graphene-based ultra-capacitors. Nano Letters, 2008, 8(10): 3498-3502.

[11] PENG S, ZENG O, GUO Y, et al. Defluoridation from aqueous solution by chitosan modified natural zeolite. Journal of Chemical Technology and Biotechnology, 2013, 88(9): 1707-1714.

[12] PURUSHOTHAMAN K K, SARAVAVAKUMAR B, BABU I M, et al. Nanostructured $\mathrm{CuO} /$ reduced graphene oxide composite for hybrid supercapacitors. RSC Advances, 2014, 4(45): 23485-23491.

[13] YANG G W, XU C L, LI H L. Electrodeposited nickel hydroxide 
on nickel foam with ultrahigh capacitance. Chem. Commun., 2008 (48): 6537-6539.

[14] WANG H, ROBINSON J T, LI X, et al. Solvothermal reduction of chemically exfoliated graphene sheets. Journal of the American Chemical Society, 2009, 131(29): 9910-9911.

[15] BORCHARDT L, OSCHATZ M, KASKEL S. Tailoring porosity in carbon materials for supercapacitor applications. Materials Horizons, 2014, 1(2): 157-168.

[16] CHMIOLA J, YUSHIN G, GOGOTSI Y, et al. Anomalous increase in carbon capacitance at pore sizes less than 1 nanometer. Science, 2006, 313(5794): 1760-1763.

[17] FENG G, CUMMINGS P T. Supercapacitor capacitance exhibits oscillatory behavior as a function of nanopore size. The Journal of Physical Chemistry Letters, 2011, 2(22): 2859-2864.

[18] WU Z S, ZHOU G, YIN L C, et al. Graphene/metal oxide composite electrode materials for energy storage. Nano Energy, 2012,
1(1): 107-131.

[19] ZHANG L L, ZHAO X S. Carbon-based materials as supercapacitor electrodes. Chemical Society Reviews, 2009, 38(9): 2520-2531.

[20] SENTHILKUMAR S T, SELVAN R K, MELO J S, et al. High performance solid-state electric double layer capacitor from redox mediated gel polymer electrolyte and renewable tamarind fruit shell derived porous carbon. ACS Applied Materials \& Interfaces, 2013, 5(21): 10541- 10550 .

[21] WANG Y, XIA Y. Hybrid aqueous energy storage cells using activated carbon and lithium-intercalated compounds. I. The $\mathrm{C} / \mathrm{LiMn}_{2} \mathrm{O}_{4}$ system. Journal of the Electrochemical Society, 2006, 153(2): A450-A454.

[22] LIU H, ZHANG W, SONG $\mathrm{H}$, et al. Tremella-like graphene/polyaniline spherical electrode material for supercapacitors. Electrochimica Acta, 2014, 146: 511-517. 